\title{
EDITORIAL
}

\section{THYROID DISORDERS IN BANGLADESH- PAST, PRESENT AND FUTURE}

\author{
ANSARI MAJ
}

Clinical experience and few laboratory tests were the only means for evaluating thyroid disorders in Bangladesh even in the early eighties of the last century ${ }^{1}$. Modern laboratory tests evolved gradually over the last 25 years making available the thyroid function tests with high sensitivity and specificity. For example old generation TSH measurements could detect only hypothyroidism not the hyper. The currently available fourth generation immunometric TSH assays in Bangladesh have sensitivity to detect TSH level upto $.02 \mathrm{mIU} / \mathrm{L}$ good enough to diagnose hyperthyroidism as well. The other thyroid function tests are also more sensitive and specific than older methods. A general belief existed among the physicians that iodine deficiency might have masked the incidence of autoimmune thyroid disorder in this part of the world.

The absolute and relative number of different thyroid disorders have changed over time as iodine deficiency disorders started to decline since early nineties of the last century. There was paucity of published reports on the spectrum of thyroid disorders in Bangladesh. The range of thyroid disorders other than iodine deficiencies was considered same in Bangladesh as in other countries of $\mathrm{Asia}^{2}$ However the relative prevalence of the different thyroid disorders was dominated by iodine deficiency disorders. Such a study published in 1995 where the author was a member reported 35\% cases of all thyroid disorders to be due to iodine deficiency as the primary etiology ${ }^{3}$. The rest were autoimmune $(26 \%)$, malignant $(2.58 \%)$ and other thyroid disorders. Earlier in 1981-82 national goiter prevalence study of Bangladesh reported overall prevalence of goiter as 10\% among Bangladeshi people.
Before that no published data could be found on thyroid disorders as mentioned clearly by Kochupillai et $\mathrm{al}^{4}$ who found no published report from Bangladesh except a comment in 1966 where one fourth the population was considered to have suffered from visible goiter in this area( Bangladesh was not a separate country then).

At the beginning of the twenty first century hypothyroidism, Graves' disease, postpartum thyroiditis and thyroid malignancies are common thyroid disorders in Bangladesh while iodine deficiency disorders are still persisting in low prevalence. It is believed that around $10 \%$ of the Bangladeshi people suffer from clinically evident thyroid disorders. Recently subclinical hypo and hyperthyroidisms are included as thyroid disorders adding another $10 \%$ population to be dysthyroid totalling $20 \%$ of the population suffering from any type of thyroid disorders. The general believe that iodine deficiency could mask the autoimmune thyroid disorder proved inconsistent by a recent study by Hasanat et $\mathrm{al}^{5}$ who found no significant change in the occurrence of autoimmune thyroid disorder due to iodine status.

Development of laboratory analysis of thyroid hormones, autoimmune markers, availability of nuclear medicine facilities, locally produced thyroid drugs and evergrowing awareness about thyroid disorders improved every aspect of thyroidology in our country. Successful implementation and maintenance of salt iodization programs followed by sharp decline of iodine deficiency disorders is a success story of Bangladesh health sector the nation can be proud of. At present extreme hypothyroidism leading to myxedema coma and madness are rarely found, thyroid nodules are detected earlier, thyroid screening during pregnancy and

1. Professor Md. Abdul Jalil Ansari, Head of the Dept. of Endocrinology, Dhaka Medical College 
neonatal periods are gaining popularity and endemic cretinism has decreased to a minimum.

In spite of all these developments thyroid related emergencies like thyrotoxic crisis, undetected congenital hypothyroidism, pregnancy loss and infertility due to thyroid disorders, inappropriate use of thyroid medications, late detection of thyroid malignancies are often the causes of significant morbidity and mortality. Treatment of Graves' ophthalmopathy is still challenging and mortality from thyrotoxic crisis is nearly $100 \%$.

Modern treatment of thyroid disorders particularly in pregnancy and neonates are highly dependent on laboratory reports requiring highly sensitive, specific and reproducible test procedures that are not widely available. We still follow the foreign laboratory reference ranges for our people that may be highly inappropriate. For example target TSH ranges applicable for pregnant women of our population are inconclusive ${ }^{6}$. Biomedical researchers are expected to conduct researches on this area.

Inappropriate treatment on unconfirmed thyroid disorders are still encountered. Laboratory reports are often not correlated with clinical status before starting treatment for thyroid disorders. Abnormal thyroid function test results in otherwise healthy persons may cause undue worry that must not prompt medication until clinically appropriate. Finding the answer to "Why do subclinical cases are being overtreated' is certainly a professional responsibility ${ }^{7}$.

Due to increased awareness and development Nuclear medicine facilities seems insufficient to the increasing demand. Diagnosing and managing thyroid disorder often needs the help of nuclear medicine that are available only in few Govt. Medical College campuses that needs expansion. People has a general phobia against radiation hazards while treating by nuclear medicine facilities. It is assuring that Nuclear Medicine Departments follow protocol to minimize radiation hazards to the patients.

Endocrinologists, physicians and surgeons interested in thyroid disorders are growing day by day. More manpower in future will certainly improve this field.

Coordinated management of thyroid disorders involves almost all the specialties of modern medicine. In Bangladesh more and more subspecialties are being developed that will certainly improve the management of thyroid disorders shortly

J Dhaka Med Coll. 2014; 23(2) : 151-152.

\section{References:}

1. Chopra IJ, Wu SY, Teco GN et al. A radioimmunoassay for measurement of 3,5,3-triiodothyronine sulfate: studies in thyroidal and nonthyroidal diseases,pregnancy, and neonatal life. J Clin Endocrinol Metab 1992; 75: 189-94

2. Weigle DS, Hooton TM, Toivola B et al. Frequency of thyroid disease among Southeast Asian primary care patients. J clin Pharm Ther 1996;21:29-35

3. Alam MN, Haq SA, Ansari MAJ et al. Spectrum of Thyroid Disorders in IPGMR, Dhaka. Bangladesh J Medicine 1995; 6: 53-58

4. Kochupillai N,Ramlingaswami V,Stanbury J B. The present status of endemic goiter as a problem of the public health-Southeast Asia In: Stanbury JB, Hetzel BS, eds. Endemic goiter and endemic cretinism-Iodine nutrition in health and disease, New delhi Wiley Eastern Limited 1985: 101-121

5. Hasanat MA, Rumi MA, Alam MN et al. Urinary iodine status and thyroid dysfunction: a Bangladesh perspective. Bangladesh Med Res Counc Bull 2004; 30: 16-24

6. Gopalakrishnan A, Menon UV. Thyroid disorders in India: An epidemiological perspective

7. Spaulding S W, Taylor PN,Iqbal A et al. Why do patients with subclinical hypothyroidism get overtreated ? Clin Thyroidol 2013; 25: 273-276 